\title{
Missed Medium-Vessel Occlusions on CT Angiography: Make It Easier ... Easily!
}

W e read with great interest the article by Fasen et al, ${ }^{1}$ which addresses an important and often underappreciated topic in radiology: diagnostic errors and, more specifically, missed large- and medium-vessel occlusions on CT angiography in patients with acute ischemic stroke. Little is known about the factors that lead to such errors, and the article adds important knowledge in this regard. Fasen et al found that a more distal occlusion location in the M2 segment in the middle cerebral artery (ie, medium-vessel occlusions $[\mathrm{MeVOs}]^{2}$ ) was missed in $82 \%$ of cases at the time of the initial CTA evaluation, and M2 occlusion location was significantly associated with the initial missing of the occlusion. M2 occlusions are increasingly targeted with endovascular thrombectomy (EVT), ${ }^{3}$ and it is, thus, crucial to accurately and reliably detect them. Fasen et al relied on axial single-phase CTA images to detect M2 occlusion, which is often challenging because the M2 segment is small in caliber and does not run horizontal to the axial plane.

Multiphase CTA (mCTA), which comprises 2 delayed phases in addition to the arterial phase with no additional contrast and minimal additional time and radiation dose, ${ }^{4}$ was successfully used for large-vessel occlusion (LVO) detection and EVT patient selection in the Endovascular Treatment for Small Core and Proximal Occlusion Ischemic Stroke (ESCAPE) and Safety and Efficacy of NA-1 in Subjects Undergoing Endovascular Thrombectomy for Stroke (ESCAPE-NA1) trials ${ }^{5,6}$ and MeVOs are much easier to catch on mCTA because a contrast hold-up is often seen in the downstream territory (Figure $A-C)$. Recent advances in mCTA postprocessing have rendered real-time generation of color-coded mCTA maps possible, which facilitates detection of LVOs and particularly MeVOs even further (Figure E). ${ }^{7}$ Lastly, mCTA can also be used to generate tissue-level perfusion maps just as CT perfusion does. In these maps, striking color changes in the downstream territory allow quick and easy detection of MeVOs (Figure $F$ ). We are convinced that the number of missed M2s would have been much lower had mCTA rather than singlephase CTA been used in the study of Fasen et al, ${ }^{1}$ and we encourage the use of mCTA in the acute stroke setting to minimize the risk of missing potential EVT target occlusions, which dramatically impacts patient management and, ultimately, worsens patient outcomes.

Disclosures: Johanna M. Ospel—UNRELATED: Grants/Grants Pending: Julia Bangerter Rhyner-Stiftung Foundation, University of Basel Research Foundation, Freiwiliige Akademische Gesellschaft Basel, Comments: research scholarships. Wu Qiu-UNRELATED: Employment: University of Calgary. Mayank GoyalRELATED: Grant: Stryker, Cerenovus*; Consulting Fee or Honorarium: Medtronic, Stryker, MicroVention, Mentice; Other: GE Healthcare, Comments: licensing agreement, systems of acute stroke diagnosis. ${ }^{\star}$ Money paid to the institution.

\section{REFERENCES}

1. Fasen B, Heijboer RJJ, Hulsmans FH, et al. CT angiography in evaluating large-vessel occlusion in acute anterior circulation ischemic stroke: factors associated with diagnostic error in clinical practice. AJNR Am J Neuroradiol 2020;41:607-11 CrossRef Medline

2. Goyal M, Ospel JM, Menon BK, et al. MeVO: the next frontier? J Neurointerv Surg 2020;12:545-47 CrossRef Medline

3. Almekhlafi M, Ospel JM, Saposnik G, et al. Endovascular treatment decisions in patients with M2 segment MCA occlusions. AJNR Am J Neuroradiol 2020;41:280-85 CrossRef Medline

4. Menon BK, d'Esterre CD, Qazi EM, et al. Multiphase CT angiography: a new tool for the imaging triage of patients with acute ischemic stroke. Radiology 2015;275:510-20 CrossRef Medline

5. Goyal M, Demchuk AM, Menon BK, et al; ESCAPE Trial Investigators. Randomized assessment of rapid endovascular treatment of ischemic stroke. N Engl J Med 2015;372:1019-30 CrossRef Medline

6. Hill MD, Goyal M, Menon BK, et al; ESCAPE-NA1 Investigators. Efficacy and safety of nerinetide for the treatment of acute ischaemic stroke (ESCAPE-NA1): a multicentre, double-blind, randomised controlled trial. Lancet 2020;395:878-87 CrossRef Medline

7. Ospel JM, Volny O, Qiu W, et al. Displaying multiphase CT angiography using a time-variant color map: practical considerations and potential applications in patients with acute stroke. AJNR Am J Neuroradiol 2020;41:200-05 CrossRef Medline 


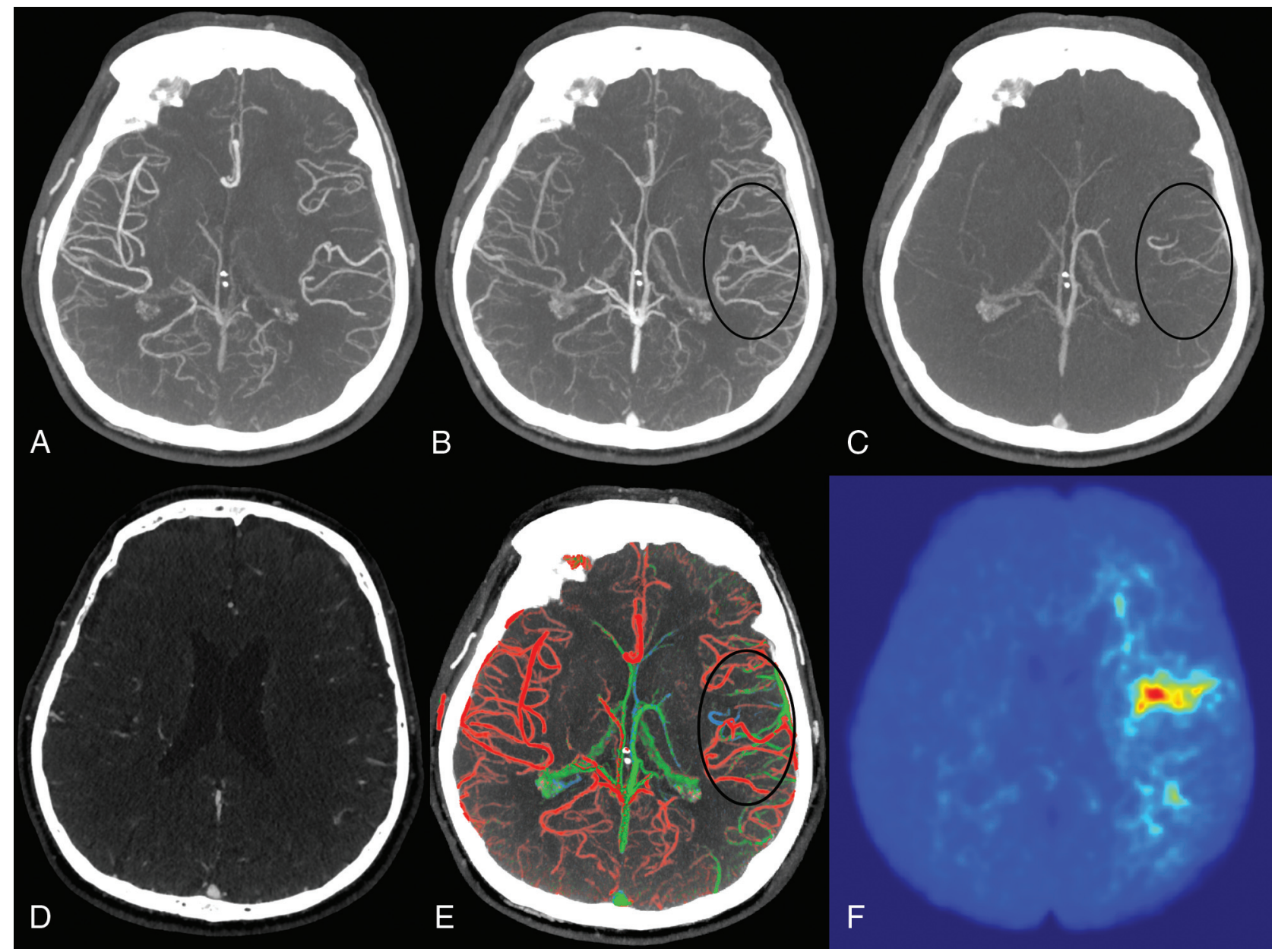

FIGURE. Patient with acute left-hemispheric symptoms (weakness of the right arm and aphasia). Multiphase CTA (arterial phase shown in A, peak-venous and late-venous phases shown in $B$ and $C$ ) shows delayed contrast washout in the left peri-Sylvian region, which raises the suspicion of a medium-vessel occlusion. On single-phase CTA (D), it is very challenging to see the occlusion or any difference in collateral filling. Color-coded time-variant mCTA summation maps facilitate the diagnosis further because the abrupt change in vessel color on the mCTA summation map is more obvious than changes in enhancement on gray-scale images (color-coded mCTA summation map is shown in $E$, the black circle highlights the color changes caused by the medium vessel occlusion), vessels with maximum enhancement in the arterial phase are shown in red, those enhancing in the peak-venous and late-venous phases are shown in green and blue). The time-to-peak map derived from mCTA ( $F)$ shows a clear change of color in the affected parenchyma and is another possibility for fast and reliable detection of MeVOs.

(D).M. Ospel

Department of Clinical Neurosciences University of Calgary

Calgary, Alberta, Canada

Department of Radiology University Hospital Basel, University of Basel Basel, Switzerland
(1)W. Qiu

Department of Clinical Neurosciences

(D) M. Goyal Departments of Clinical Neurosciences and Radiology University of Calgary Calgary, Alberta, Canada 